DOI: http://dx.doi.org/10.12775/szhf.2014.021

\author{
Anita Pacholik-Żuromska
}

\title{
Dimensionen des Verstehens
}

\author{
Einleitung
}

In der gegenwärtigen Philosophie des Geistes, die sich schon lange mit dem Mind-Body-Problem und mit der Frage, ob Maschinen denken können, beschäftigt hat, gibt es-deutliche Trends, die den Physikalismus überwinden, um ein neues Land zu entdecken. Dieses neue Land ist der Mensch, der als biologisches Wesen im Zusammenhang mit seiner Umgebung betrachtet wird. Seit der Zeit von Rene Descartes, der eigentlich „der Vater des KörperGeist-Problems“ genannt werden kann, hat sich bis zum heutigen Tage viel geändert. Seiner Meinung nach sind Körper und Geist ontologisch unterschiedliche und voneinander unabhängige Substanzen. Beide gehören aber zum Menschen. Epistemologisch stellte Cogito (Ich denke) die feste und einzige Grundlage des sicheren Wissens dar. Nach Descartes kann man an allem, auch an der Existenz der Welt, zweifeln. Man kann aber nicht in Frage stellen, dass man denkt. Dubito, ergo sum - sagt der Philosoph. Dieses Wissen ist möglich dank dem unmittelbaren Zugang zu den eigenen mentalen $\mathrm{Zu}$ ständen. Wir erfahren uns selbst in einem Akt der Introspektion, die als eine direkte Wahrnehmung der psychischen Phänomene definiert wird. Cogito ist also die letzte Instanz, die uns eine sichere Erkenntnis garantiert. Das bedeu- 
tete auch, dass nur Menschen fähig sind, ihre Existenz zu verstehen bzw. zu verstehen, dass sie existieren.

Descartes hatte aber ein großes Problem damit zu erklären, wie der Geist den Körper beeinflusst. Obwohl er ohne Zweifel die Konstitution des Menschenseins in res cogitans sah, wobei der Körper nur eine Rolle des Seelenträgers spielte, musste er sich sehr darum bemühen, zu erklären, wie der Körper die Befehle vom Geist empfängt und umsetzt. Endlich hatte er den Ort für die Seele in der Zirbeldrüse gefunden, die als das erste Korrelat des Bewusstseins gelten kann.

In den gegenwärtigen Zeiten ist die Geist-Körper-Frage völlig umgekehrt. Man zweifelt nicht, dass die Grundlage der mentalen Zustände physisch ist. Das Problem liegt nun aber darin, wie das Mentale entsteht. Es ist besonders interessant, welche biophysiologischen Prozesse für die psychischen Phänomene verantwortlich sind. Und ob es nötig ist, die bisherigen typisch menschlichen mentalen Kapazitäten, wie Verstehen, Rationalisieren, Intendieren usw. neu zu definieren.

Um die Jahrhundertwende entwickelte sich in Amerika eine neue Richtung innerhalb des Psychologie-Behaviorismus, die auch als „radikaler Antimentalismus" bezeichnet wird. Nach dieser naturalistischen Stellung müssen alle mentalistischen Aussagen, also alle Feststellungen, die nicht unmittelbar anhand des beobachteten Verhaltens einer Person überprüft werden können, als fiktiv und somit als wissenschaftlich unzulässig gelten. Dieser Grundgedanke leitete den Beginn der Naturalisierung des Geistes ein. Danach wurde es immer radikaler. Heutzutage ändert die Hirnforschung unser Menschenbild mit weitgehenden, sogar ethischen Konsequenzen ${ }^{1}$. Die sich rasch entwickelnden Neurowissenschaften geben fortwährend neue Hinweise darauf, dass Denken, Subjektsein und Willensfreiheit eine Illusion der menschlichen Spezies sind.

In diesem Artikel wird es versucht, die menschlichen mentalen Kapazitäten im Bezug auf die Frage des Verstehens im Licht der neuesten Erkenntnisse der Hirnforschung zu überdenken. Es wird die These aufgestellt, dass es nicht ausreichend ist, das Verstehen auf die neuronale Ebene zurückzuführen, um es zu erklären. Das Verstehen hat verschiedene Dimensionen, die sich erst im Aspekt der Interpretation, Kommunikation und sozialen Interaktionen

${ }^{1}$ Vgl. Ein Frontalangriff auf unser Selbstverständnis und unsere Menschenwürde. Diskussion mit Wolf Singer und Thomas Metzinger, Gehirn \& Geist, Nr 4, 2002, S. 14-17. 
enthüllen. Anders gesagt, das Paradigma der Neurowissenschaften ist für das Verstehen explanativ unvollständig.

\section{Verstehen auf der subpersonalen Ebene}

Mit den „neusten Erkenntnissen der Hirnforschung“ meinen wir hier die herausragende Entdeckung der Spiegelneuronen vom Forschungsteam Giacomo Rizzolattis und Vittorio Galleses. Das Spiegelneuronensystem befindet sich im Feld F5 des Großhirns (ventraler prämotorischer Kortex). Seine Rolle ist das beobachtete Verhalten im Gehirn zu simulieren ohne es auszuführen. Die Standardexperimente, die eine Aktivierung der Spiegelneuronen zeigen, bestehen darin, dass man einem Subjekt (Makakaffe) ein Objekt zeigt (Nüsse, Banane usw.), das der Affe greifen soll. Wenn der Affe nach dem Objekt greift, feuern bestimmte Nervenzellen im Kortex. Im zweiten Teil des Experiments beobachtet der Affe die Bewegung eines anderen Subjektes (z.B. einer Person des Forschungsteams). Während der Affe zuschaut, wie jemand anderes das Objekt greift, feuern dieselben Nervenzellen wie in der Situation, in welcher er selbst handeln würde. Ähnliche Experimente haben gezeigt, dass auch Menschen ein Spiegelneuronensystem haben.

Diese Ergebnisse haben zur Aufstellung der These geführt, dass die Spiegelneuronen sehr wichtig für den Verstehensprozess sind. Das wurde unter anderem von Rizzolatti geprüft und steht nicht mehr im Fokus der Diskussion. Die Frage ist aber, welche Rolle die Spiegelneuronen im Verstehen spielen? Wir nehmen also an, dass die Spiegelneuronen für das Verstehen des Verhaltens grundlegend sind. Wir fragen darüber hinaus, ob sich eine gerade Linie von der neuronalen Ebene bis zur personalen Ebene in jeden Fall des Verstehens führen lässt.

Es ist schwer die funktionale Rolle der Spiegelneuronen im Allgemeinen zu beschreiben. Rizzolatti weist auf die zwei Hypothesen hin, die er jedoch für falsch hält ${ }^{2}$. Kurz gesagt, die Rolle der Spiegelneuronen ist nicht ausreichend erklärt, wenn man sagt, dass sie für das „bereit sein“ verantwortlich sind. Eine bessere Erklärung gibt Marc Jeannerod an, wenn er sagt, dass sie eine bestimmte Funktion in der Nachahmung (Resonanz) erfüllen ${ }^{3}$. Damit

\footnotetext{
${ }^{2}$ Giacomo Rizzolatti, Corrado Sinigaglia, Empathie und Spiegelneurone. Die biologische Basis des Mitgefühls, Suhrkamp Verlag, Frankfurt am Main 2008, S. $103 \mathrm{ff}$.

${ }^{3}$ Marc Jeannerod, The Representing Brain: neural correlates of motor intention and imaginery, [in:] Behavioral Brain Sciences, 17(1994), S. 187-245.
} 
sind sie wichtig für das Lernen. Die Spiegelneuronen von F5 und des Komplexes PF-PFG haben aber evolutionär noch eine ursprünglichere Funktion als die Nachahmung, nämlich Erkennen und Verstehen der Bedeutung von motorischen Ereignissen, also die Akte der anderen ${ }^{4}$. Jedoch ist hier das Verstehen sehr einfach definiert: Es ist „die unmittelbare Fähigkeit in den beobachteten motorischen Ereignissen einen bestimmten Typ von Akt zu erkennen, der gekennzeichnet ist durch eine spezifische Modalität der Interaktion mit Objekten, die Fähigkeit, diesen Typ von anderen zu unterscheiden und eine solche Information gegebenenfalls zu nutzen um auf die angemessenste Weise zu reagieren “5. D.h., der visuelle Reiz wird vom entsprechenden motorischen Akt kodiert, also auf bestimmte Weise gespeichert. Das bedeutet, dass ein Affe ein motorisches Ereignis wahrnimmt und als einen bestimmten Typ der Aktion „versteht“.

Das Verstehen begreift Rizzolatti nicht in einer reflexiven Form, sondern pragmatisch. Es scheint, dass der Terminus „,behavioristisch“ mit Rücksicht auf den Zusammenhang Reiz-Reaktion hier besser passt. Für die notwendige und hinreichende Bedingung des Verstehens der Akte anderer hält er die Tatsache, dass das Individuum die intentionale Bedeutung seiner eigenen Akte kennt und das motorische Wissen besitzt, das es aus der Bestätigung ihrer möglichen Folgen ableitet ${ }^{6}$.

Es ist unstreitig, dass das Verstehen den Prozess des Denkens betrifft. Man schreibt es sowohl Menschen als auch anderen Tieren zu. Sie agieren als ob sie verstünden, wenn sie zum Beispiel auf Befehle reagieren. Das passiert, weil man dieses Verhalten als intentional interpretiert. Man sollte aber zwischen Reaktion und Intention unterscheiden. Es ist ein Fehler von einer Reaktion auf eine Intention zu schließen. Auf diese Weise könnte man Intention sogar den Thermostaten zuschreiben, wenn sie auch auf Temperatur entsprechend reagieren - so lautet auch der bekannte Kommentar von Daniel Dennett. Eine richtige Reaktion auf einen Reiz ist keine Intentionalität. Aber das Verstehen eigener Reaktionen ist intentional. Das Verstehen der Reaktionen bedeutet die Interpretation der Reaktionen. Auf der subpersonalen Ebene sollte man also vom Verstehen in einem engen Sinn als eine Fähigkeit der Verbindung von bestimmten visuellen Reizen mit ihren Konsequenzen, also von bestimmten Inputs mit bestimmten Outputs sprechen.

${ }^{4}$ Ebenda, S. 106.

${ }^{5}$ Ebenda, S. 106-107.

${ }^{6}$ Ebenda, S. 114. 
Manche Forscher, die sich auf Spiegelneuronen beziehen, schließen aus, dass der Verstehensprozess sich nur auf die neuronale Ebene reduzieren lässt. Peter Gärdenfors unterscheidet zwischen vier Stufen des Verstehens ${ }^{7}$ :

1. Verstehen von Emotionen

2. Verstehen der Aufmerksamkeit

3. Verstehen von Intentionen

4. Verstehen des Wissens von Anderen

Elisabeth Patcherie und Jerome Dokic unterscheiden zwischen vier Ebenen des Wissens ${ }^{8}$ :

1. Visuelles Wissen

2. Motorisches Wissen

3. Agentives Wissen

4. Meta-repräsentationales Wissen

In beiden Fällen lassen sich die drei ersten Arten des Verstehens bzw. Wissens auf Nervenprozesse, d.h. auf eine Stimulation der Spiegelneuronen reduzieren. Im Fall des meta-repräsentationalen Wissens und des Verstehens des Wissens von anderen gibt es hier auch eine Verbindung, aber sie ist unterbrochen, weil gerade hier das Körper-Geist-Problem entsteht: Wie kann man von der physischen zur mentalen Ebene übergehen? Der vierte Fall des Verstehens ist deswegen besonders interessant. In diesem Artikel wird er am Beispiel des rationalen und des hermeneutischen Denkens dargestellt. Es sollte klar sein, dass die Reduktion zurück auf die subpersonale Ebene der holistisch angenommenen, höheren mentalen Zustände, die die sogenannten Geist-Theorien bilden, in diesem Fall eigentlich nicht möglich ist.

\section{Verstehen als rationales Denken}

In diesem Kapitel wird es die These aufgestellt, dass das Verstehen als rationales Denken von der Sprache abhängt. Anders gesagt, mit der Entwicklung der Sprache entwickelte sich auch die Fähigkeit zu Verstehen, besonders wenn es um die Zuschreibung der mentalen Zustände an andere geht.

Das Verstehen in diesem Sinn definiert Mathias Günther, der in seiner Deutung an Dilthey anknüpft:

\footnotetext{
${ }^{7}$ Peter Gärdenfors How Homo Became Sapiens: On the Evolution of Thinking, Oxford University Press, 2003.

${ }^{8}$ Elisabeth Patcherie, Jerome Dokic, From mirror neurons to joint actions, Cognitive Systems Research, 7(2006), S. 101-112.
} 
Das Verstehen anderer Menschen, ihre psychologische Beschreibung und die Erklärung ihrer Handlungen erfolgt durch Übertragung des unmittelbar über die eigenen psychischen Zusammenhänge Gewussten: „[I]n das Innere anderer Individuen leuchten wir mit dem Lichte der Analogie“. „Verstehen ist die Beschreibung eines spezifischen Strukturzusammenhangs der Handlungen, psychische Zustände und äußere Einflüsse auf interpretanden-spezifische Weise zusammenordnet, wobei, die Elemente des verstehenden Beschreibens aus dem introspektiv bekannten psychischen Zusammenhang entnommen werden'.

Was untrennbar von dem Verstehen ist, ist die Intentionalität, also eine Ausrichtung auf ein Objekt. Das Verstehen als ein höherer Denkprozess zeichnet sich durch seine propositionale Form aus. Im Verstehen wird ein Urteil Kraft seiner Bedeutung akzeptiert oder abgelehnt. Anders gesagt, es wird mit einer Begründung für falsch oder wahr gehalten. Die auf diese Weise charakterisierten mentalen Zustände werden nach der Art ihres Inhaltes propositionale Einstellungen genannt. Das sind Relationen zwischen Individuen und Propositionen (Sätzen), wobei manche Philosophen, wie Jerry Fodor, ein drittes Argument dazu einführen - die Art des Gegebenseins. Nach ihm sind die Arten des Gegebenseins Sätze (in der Sprache des Denkens [mentalese]), und diese Sätze sind nicht nur durch den Inhalt der Proposition, sondern auch durch die Syntax individuiert. Die Hypothese der Sprache des Denkens ist besonders nützlich, wenn man annehmen will, dass Verstehen und Erkennen außerhalb der natürlichen Sprachen (Muttersprachen) möglich sind.

Das Verstehen als rationales Denken ist für einen gelungenen Kommunikationsakt notwendig. Es gibt viele Theorien, die die Bedingungen aufzählen, unter denen ein Kommunikationsakt ausgeführt werden kann. Vor allem gelingt ein Kommunikationsakt, wenn Sender und Empfänger sich untereinander verstehen. Dazu müssen sie im Allgemeinen die Bedeutung der Wörter kennen und die Sätze korrekt aufbauen können.

Wenn Sender und Empfänger ihre Äußerungen den Regeln entsprechend aufbauen, dann ist es ein Hinweis darauf, dass sie die Sprache verstehen. Dann entsteht eine Relation, in der sich ein Sender auf die Welt bezieht. Man sollte aber fragen, ob das Verstehen der Sprache schon das Verstehen des Gesprächspartners mit sich bringt? Natürlich nicht. Dass ich meine Äußerun-

\footnotetext{
${ }^{9}$ Mathias Günther, Prinzipien der Interpretation. Rationalität und Wahrheit. Donald Davidson und die Grundlagen einer philosophischen Theorie des Verstehens, Mentis, Paderborn 2002, S. 289.
} 
gen verstehe, bedeutet noch nicht, dass ich die Äußerungen von jemandem anderen verstehe.

Die Weise des Verstehens von vielen, vielleicht sogar allen Sätzen der Alltagssprache hängt von den Umständen ab, in den sie gebraucht werden: davon, wer spricht, vom Moment der Äußerung, von dem Gesprächspartner, usw $^{10}$.

In ihren Äußerungen beziehen sich Sender und Empfänger auf die Welt, aber auch auf die Äußerungen des Gesprächspartners. Es entsteht eine Triangulation des Verstehensprozess, wobei das Dreieck vom Sender, Empfänger und der Welt gebildet wird. Wie Donald Davidson in seiner Theorie der radikalen Interpretation meint, der Empfänger, den er als Interpreten bezeichnet, konstruiert Hypothesen aufgrund des Verhaltens des Senders, um seine Äußerungen zu verstehen und konfrontiert ständig diese Hypothesen mit der Welt ${ }^{11}$.

Es ist also sehr wichtig, sowohl das Verhalten als auch die Äußerung im Zusammenhang mit einer bestimmten Situation zu interpretieren. Dazu zählt ebenfalls die physische und soziale Umgebung des Subjekts. Aber auch seine mentale Kondition. Der Kontext setzt sich also aus verschiedenen Faktoren zusammen. Die Bedeutung des sprachlichen und körperlichen Verhaltens (wobei die Welt als Referent im Allgemeinen verstanden wird) wird dabei als eine komplexe Relation im Kontext der Situationen verstanden.

Eine interessante Herausforderung für das hermeneutische Denken stellt der Idiolekt dar. Als Idiolekt wird eine eigene, persönliche, private Sprache, die nur einem Individuum zugeschrieben wird, definiert. Dieses Sprachphänomen stellt unter anderem einen interessanten Themenbereich der Semantik dar. Es geht hier nämlich um eine Beantwortung der folgenden Fragen: Wie entsteht die Relation zwischen Wort und Objekt? Was determiniert die Bedeutung? Wäre eine Interpretation möglich, wenn jeder nur Idiolekt sprechen würde? Die oben genannten Probleme sind auch in der Sprachphilosophie (z.B. bei G. Frege, L. Wittgenstein) vorhanden. Die philosophischen Aspekte der Idiolektsproblematik spiegelt ein Beispiel des Kommunikationsproblems wider, das im Buch Alice hinter den Spiegeln vorzufinden ist, wo das anthropomorphisierte Ei Humpty Dupty seine eigene Sprache im Dialog mit Alice benutzte. Der Dialog führt nirgendwohin, weil dasselbe Wort jederzeit

\footnotetext{
${ }^{10}$ Marek Tokarz, Elementy pragmatyki logicznej, PWN, Warszawa 1993, S. $113 \mathrm{ff}$.

${ }^{11}$ Vgl. Donald Davidson, The Structure and Content of Truth, The Journal of Philosophy, 87 (1990), Nr. 6, S. 297-328.
} 
ein anderes Objekt bezeichnet. Das bedeutet, dass die erwünschte Stufe des gemeinsamen Verstehens nicht zu erreichen ist. In Konsequenz dessen ist das Gespräch sinnlos, was wiederum der These von Ludwig Wittgenstein widerspricht, dass die Bedeutung des Wortes seine Anwendung ist.

Wenn du einmal weißt, was das Wort bezeichnet, verstehst du es, kennst seine ganze Anwendung ${ }^{12}$.

Das betrifft aber den Idiolekt nicht. Ein Text ist ein geöffnetes System und seine vollständige Interpretation ist erst dann möglich, wenn zuerst festgestellt wurde, auf welcher Sprachebene sich die bestimmten Sätze befinden. Ist das die Metasprache oder die Objektsprache? Davon hängt der Referent ab. Entweder ist das ein anderer Satz oder ein Objekt in der Welt. Die Schwierigkeiten in der Erkennung des Referenten, also der Bedeutung, verursachen Verstehensschwierigkeiten.

Wie schon gesagt wurde, man hat es im Kommunikationsakt mit einem hermeneutischen Rätsel zu tun, weil es nicht einfach ist, die genaue Bedeutung der Äußerungen der Gesprächspartner zu entdecken. Für eine Interpretation ist charakteristisch, dass sie etwas enthüllt, manchmal sogar entlarvt. Dieses Enthüllen ist im Fall der Dialoge auch nötig. Um den Sinn einer Äußerung zu verstehen, muss man zuerst begreifen, worauf sich der Gesprächspartner bezieht. In der Pragmatik der Sprache geht es immer um die Relation zwischen dem Sprecher und der Welt. Es ist nicht einfach die Intentionen des Sprechers ohne zusätzliche Informationen zu verstehen, umso mehr, wenn Äußerungen auf Ellipse, Amphibolie und Äquivokation basieren.

Der Kernpunkt scheint zu sein, dass zum Verständnis konkreter Handlungen ein Wissen um die aktuellen Bedürfnisse und Absichten des jeweiligen Handelnden notwendig ist. Mit anderen Worten: praktische Aktionen werden durch aktuelle Ziele des Handelnden determiniert ${ }^{13}$.

Normalerweise sollen die Bezugsrelationen - Referenzen - zwischen sprachlichen Zeichen und ihren Referenten richtig bestimmt werden, wie im Fall:

${ }^{12}$ Ludwig Wittgenstein, Philosophische Untersuchungen, Suhrkamp Verlag, Frankfurt am Main 2003, S. 153.

${ }^{13}$ Max Urchs, Maschine, Körper, Geist. Eine Einführung in die Kognitionswissenschaft, Vittorio Klostermann, Frankfurt am Main 2002, S. 7. 
"der Stuhl” der Stuhl

Das Wort „der Stuhl“ steht in einer Bezugsrelation zum Stuhl (Objekt in der Welt). Analog steht S.2. steht in der Bezugsrelation zu S.1.

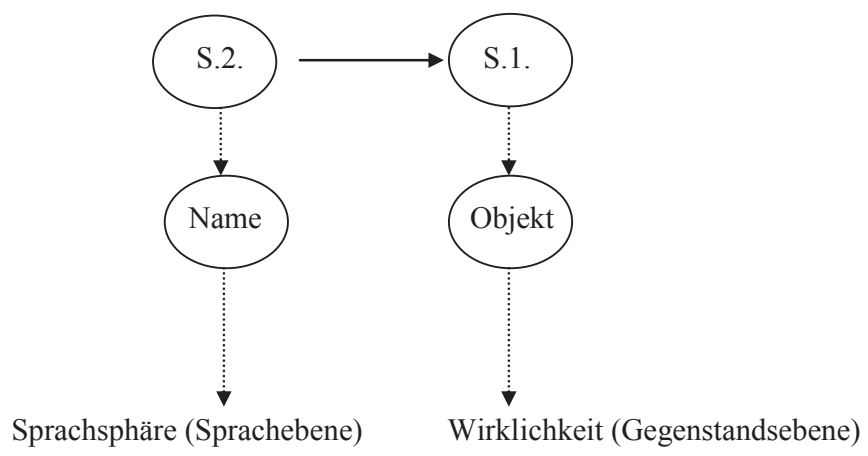

Das Diagramm soll zeigen, dass eine Aussage S.2. wahr ist, wenn es mit der Welt korrespondiert. Das Bezugsobjekt S.1. muss als ein Element (Sachverhalt) der Wirklichkeit unter S.2. fallen. Diese einfachen Relationen gibt es in alltäglichen Gesprächen selten.

\section{Verstehen als hermeneutisches Denken}

Ein besonderes Beispiel der menschlichen Denkfähigkeiten ist das hermeneutische Denken, worunter ästhetisches und ethisches Denken fallen. Das ist eine besondere Art des höheren Verstehens, wo man es mit einer indirekten Kommunikation zu tun hat, das heißt, wenn verschiedene (behaviorale, sprachliche) Zeichen symbolisch sind und Expressionsmittel wie Literatur und Kunst benutzt werden. Die sinnvolle Bearbeitung derartigen Daten verlangt eine Interpretation im Verstehensprozess.

Was ist nämlich eine Interpretation? Diese Frage gehört zu dem Bereich der Hermeneutik: der Lehre von Verstehen, Deuten, Auslegen. Einen Text oder ein Kunstwerk zu interpretieren, heißt also es zu verstehen und zu erklären. „Auslegen ist [...] der Vollzug des Verstehens selbst [.]“14

Nach Hans-Georg Gadamer geht das Verstehen der Interpretation nicht schrittweise voran, sondern es ist ein gleichzeitiger Prozess. Das Verstehen und das Interpretieren sind dasselbe. Bei manchen Texten muss man die Ent-

${ }^{14}$ Hans-Georg Gadamer, Wahrheit und Methode, 2. Aufl., J.C.B. Mohr (Paul Siebeck), Tübingen 1960, S. 375. 
stehungsumstände des Werkes kennen, um es verstehen zu können. Dieser Kontext wird oft durch die Gesellschaftsverhältnisse bestimmt.

Obwohl Kunst einen überzeitlichen Wert haben kann, der sowohl für die Zeitgenossen vom Künstler, als auch für die Nachfolger ihre Aktualität nicht verliert, hängt sie immer mit Raum und Zeit zusammen, in denen sie entstanden ist. Raum und Zeit bilden die Rahmen des Werkes: sowohl einer Skulptur, eines Gemäldes als auch einer Erzählung. Man darf sogar sagen: Die sittlichen-politischen-wissenschaftlichen Verhältnisse einer Epoche geben die bestimmten Interpretationswerkzeuge in die Hand des Interpreten. Es ist also unmöglich, bei der Deutung der Literaturwerke von den sie begleitenden Gedankenströmungen und Ereignissen abzusehen .

Mit der Ästhetik wurden sehr lange die gesellschaftlichen Sitten assoziiert. Die Nebeneinanderstellung der beiden Begriffe "Sitte“ und „Ästhetik“ hat ihre Begründung in der Kunst- und Literaturgeschichte. Die ästhetische Funktion der bestimmten Motive der Literatur beruht darauf, dass sie oft als Stilmittel betrachtet werden, so wie Metapher, Oxymoron, Parabel usw. Gleichzeitig erfüllen sie eine ethische Funktion, indem sie die gesellschaftlichen Beziehungen - dabei auch die Sitten - beschreiben und kritisieren. Damit dienen sie auch als Belehrung. Die Verbindung zwischen Sitte, die zum Bereich der ethischen Untersuchungen gehört, und Ästhetik, die in ihrem Umfang auch die Hermeneutik, also die Interpretationslehre enthält, braucht eine ausführlichere Erklärung.

Der Begriff „Ästhetik“ wurde von Alexander Gottlieb Baumgarten im Jahre 1735 eingeführt. Damit begründete er Ästhetik als eine eigenständige Disziplin, die als eine Theorie des analogischen Denkens und als die Lehre der sinnlichen Wahrnehmung definiert wurde ${ }^{15}$. Natürlich wird die Ästhetik vor allem mit der Schönheitstheorie assoziiert, was auch stimmt, aber diese Bedeutung ist zu eng. „Ästhetik“ bezog sich ursprünglich auf das altgriechische Wort aisthetikos - 'der Erfühlende'. Wenn Ästhetik nur die Lehre der Schönheit wäre, würde sie „Kallistik“ heißen (von kallos - 'schön'). Aber um etwas als schön zu empfinden, muss man das betrachtete Objekt erfahren, also sinnlich wahrnehmen. Das ist die erste Bedingung des ästhetischen Urteils. Die zweite Bedingung ist der entsprechende Geschmackssinn. Dieser Geschmack (je nach Theorie) kann entweder individuell oder objektiv bedingt

${ }^{15}$ Alexander Gottlieb Baumgarten, Aesthetica, [in:] Theoretische Ästhetik. Die grundlegenden Abschnitte aus der „Aesthetica“, Lateinisch-Deutsch, übers. und hrsg. von Hans Rudolf Schweizer. Philosophische Bibliothek, Band 351. Meiner, Hamburg 1983, \$1. 
sein. Auf jeden Fall ist es aber eine besondere Urteilskraft, die den Menschen ermöglicht, das Schöne von dem Hässlichen zu unterscheiden. Aus diesem Grund fragen manche Philosophen, die der Philosophie von Immanuel Kant folgen, nicht nach den Ursachen der Verschiedenheit des Geschmacks, sondern nach einer Möglichkeit des Urteils selbst ${ }^{16}$. „Denn der Geschmack muss ein Selbsteigenes Vermögen sein ${ }^{\text {“17 }}$.

Der Begriff „Ethik“ wurde von Aristoteles eingeführt und bezeichnete die wissenschaftliche Beschäftigung mit Sitten, Gebräuchen und Gewohnheiten. Im Allgemeinen beschäftigt sich die Ethik mit der Moral, indem sie Kriterien für gutes und schlechtes Handeln und für die Bewertung der Motive und Folgen von bestimmten Taten aufstellt. Im Rahmen der Ethik versucht man die Hauptfrage: „Was soll ich tun?" ${ }^{“ 18}$ zu beantworten. Auch Ethik ist mit dem Begriff „Geschmack“ verbunden.

So ist Geschmack zwar gewiss nicht die Grundlage, wohl aber die höchste Vollendung des Sittlichen Urteils. Wem das Unrechte gegen den Geschmack geht, dessen Sicherheit im Annehmen des Guten und Verwerfen des Schlechten ist die höchste [.]

Es ist dies eine humanistische und damit am Ende griechische Komponente, die innerhalb der durch das Christentum bestimmten Moralphilosophie wirksam wird. Die griechische Ethik - die Maßethik der Pythagoreer und Platos, die Ethik der Mesotes, die Aristoteles geschaffen hat - ist in einem tiefen und umfassenden Sinne eine Ethik des guten Geschmacks ${ }^{19}$.

Die Relation zwischen Ethik und Ästhetik im oben genannten Sinn kann kurz und knapp so formuliert werden: Was gut ist, ist auch schön.

Auch wir verbinden mit dem Begriff des „Schönen“ unter Umständen, dass etwas in der Öffentlichkeit anerkannt ist durch Brauch und Sitte oder was immer sonst; dass es - wie wir sagen - sich sehen lassen kann und auf das Ansehen hin determiniert ist ${ }^{20}$.

${ }^{16}$ Siehe: Immanuel Kant, Kritik der Urteilskraft, Hrsg. von Wilhelm Weischedel, Suhrkamp, Frankfurt am Main 2004.

${ }^{17}$ Immanuel Kant, Kritik der Urteilskraft, $\$ 17$. Zitat nach: Hans-Georg Gadamer, Wahrheit und Methode, 2. Aufl., J.C.B. Mohr (Paul Siebeck), Tübingen 1960, S. 40.

${ }^{18}$ Immanuel Kant, Kritik der praktischen Vernunft, Hrsg. von Wilhelm Weischedel, Suhrkamp, Frankfurt am Main 2004.

${ }^{19}$ Hans-Georg Gadamer, Wahrheit und Methode, S. 37.

${ }^{20}$ Hans-Georg Gadamer, Die Aktualität des Schönen. Kunst als Spiel, Symbol und Fest, Philipp Reclam, Stuttgart 1989, S. 17. 
Aber es gibt noch einen anderen Aspekt dieses Zusammenhangs, sobald die ästhetische Funktion des Kunstwerks auch eine ethische Rolle spielt. Das passiert, wenn das ästhetisch betrachtete Objekt gleichzeitig eine ethische Einstellung der Zuschauer erweckt. Diese Erscheinung ist zum Beispiel in der Kirchenarchitektur sehr gut sichtbar. Die verzerrten Gesichter der Maskarone dienten nicht nur als Dekor bei Gebäuden. Sie stellten auch eine Warnung vor bösen Dämonen dar, die die Sünder bestimmt in der Hölle treffen. Hässliche und deformierte Gesichter sind auch in der Malerei, besonders in den Werken mit religiöser Thematik, präsent. Die wahrgenommenen ästhetischen Elemente sollen also auch einen Anstoß zum ethischen Nachdenken darstellen.

Um dem Kunstwerk irgendwelche Bedeutung zuschreiben zu können, muss man es zuerst verstehen.

Wie immer das Nachfühlende Verstehen näher zu charakterisieren sein mag, es soll jeden falls eine Methode darstellen, um zu geeigneten Erkenntnissen zu gelangen, sei es zu einem Wissen über die in einem konkretem Fall vorliegenden psychischen Prozesse und geistigen Inhalte, sei es zu Einsichten in allgemeine Zusammenhänge zwischen seelisch-geistigen Vorgängen ${ }^{21}$.

Das bedeutet, dass in der Interpretation eines Kunstwerkes ein Erkenntniserfolg nur in einem Einfühlungsprozess möglich ist.

Bei der Interpretation eines Literaturwerkes hat der Leser es hier mit einer besonderen Welt zu tun, die nur auf der propositionalen Ebene der rein intentionalen Objekte bzw. der inneren Objekte des Geistes oder Repräsentationen untersucht werden sollte. Auf dieser Ebene werden die Relationen zwischen Objekten der inneren Welt in der Metasprache geäußert, denn ein literarisches Werk stellt eine fiktive Realität dar, die ein Modell einer bestimmten Konstellation von Sachverhalten bildet. Diese Sachverhalte existieren nicht wirklich und man fragt, worauf man sich in diesem Fall bezieht. Darf man etwas über die Wahrheit der fiktiven Situationen aussagen? Lassen sie sich auf diese Weise beurteilen? Das ist eine der wichtigen sprachphilosophischen Fragen: Was denotieren die Sätze, die nur Sinn haben?22

Doch bauen die Sätze mit ihrem Inhalt eine besondere Struktur der fiktiven Welt auf, in der sich alles so wie auf einer Bühne vor den Augen der Zuschau-

${ }^{21}$ Wolfgang Stegmüller, Die Sogenannte Methode des Verstehens, [in:] Lust an der Erkenntnis: Die Philosophie des 20. Jahrhunderts, Volker Spierling (Hrsg.), Serie Piper, München 1986.

${ }^{22}$ Vgl. z.B. Bertrand Russel, On Denoting, Mind 14 (1905), S. 479-493. 
er abspielt. Nach Roman Ingarden wird diese innere Welt eines literarischen Werkes von Dingen, Menschen und Relationen gestaltet, aber diese Entitäten gehören zu einer speziellen Kategorie, weil das Werk rein intentional ist ${ }^{23}$. Anders gesagt, besteht diese fiktive Welt, wie die Realität, aus Subjekten, Objekten und Beziehungen, die aber in einer zeit-räumlichen Dimension nicht verortet werden können.

Der Geist begreift, dass er es mit einer Fiktion zu tun hat. Das heißt, dass auf einer höheren Stufe des Denkens die Grenze zwischen Realität und Imagination bekannt ist. Aber die Emotionen, die der Zuschauer z. B. eines griechischen Dramas erlebt, sind wahr. Katharsis ist möglich dank einer Mimesis, also dank einer Abbildung von wirklichen Situationen. Dieses emotionale Einfühlen ist dank der entsprechenden neuronalen Prozesse natürlich und eben dank des Spiegelneuronensystems möglich. Die Zuschauer wissen, was der Schauspieler auf der Bühne fühlt. In der Tat sehen sie keinen Schauspieler sondern einen Menschen wie sie. Sie stellen sich vor, was sie an seiner Stelle tun würden. In diesem Punkt, im Moment dieser Vorstellung endet die Aufgabe des subpersonalen „Verstehens" und es beginnt die Rolle des hermeneutischen Denkens.

\section{Zusammenfassung}

Wie bereits gesagt wurde, die Frage des Verstehens sollte auf vielfacher Ebene entschieden werden. Es ist nicht klar ersichtlich, dass der Prozess sich auf die neuronale Ebene zurückführen lässt. Dieses Problem ist noch nicht einmal von der Ontologie abhängig. Die Anhänger des Materialismus gestehen auch ein, dass das Verstehen nicht nur von einem Training des neuronalen Netzes abhängt. Das Verstehen kann also eine bestimmte Disposition zum Reagieren sein, aber in den komplizierten Fällen, wo es um die Interpretation eines Kunstwerkes oder das Verstehen von Implikaturen geht, ist diese Erklärung zu schwach.

Natürlich basieren die mentalen Zustände auf der physischen Struktur des Gehirns. Es gelingt aber nicht, die Relationen zwischen den einzelnen mentalen Zuständen, z.B. eine Schlussfolgerung von der ersten Überzeugung über die Nächste bis zu einem Schluss, auf Relationen zwischen neuronalen

\footnotetext{
${ }^{23}$ Roman Ingarden, Das literarische Kunstwerk. Eine Untersuchung aus dem Grenzgebiet der Ontologie, Logik und Literaturwissenschaft, Halle 1931, Max Niemeyer und Roman Ingarden Vom Erkennen des literarischen Kunstwerks, Tübingen 1968, Max Niemeyer.
} 
Zuständen zu reduzieren. Deswegen ist es notwendig und das ist auch die Aufgabe der Philosophen, deutlich zu unterstreichen, dass der Verstehensprozess multidimensional ist.

\section{Literaturverzeichnis}

Baumgarten A. G., 1983, Aesthetica, [in:] Theoretische Ästhetik. Die grundlegenden Abschnitte aus der „Aesthetica“, Lateinisch-Deutsch, übers. und hrsg. von Hans Rudolf Schweizer, Philosophische Bibliothek, Band 35, Meiner, Hamburg.

Davidson D., 1990, The Structure and Content of Truth, The Journal of Philosophy, 87(1990) Nr. 6.

Gadamer H. G., 1989, Die Aktualität des Schönen. Kunst als Spiel, Symbol und Fest, Philipp Reclam, Stuttgart.

Gadamer H. G., 1960, Wahrheit und Methode, 2. Aufl., J.C.B. Mohr (Paul Siebeck), Tübingen.

Gärdenfors P., 2003, How Homo Became Sapiens: On the Evolution of Thinking, Oxford University Press.

Günther M., 2002, Prinzipien der Interpretation. Rationalität und Wahrheit. Donald Davidson und die Grundlagen einer philosophischen Theorie des Verstehens, Mentis, Paderborn.

Jeannerod M., 1994, The Representing Brain: neural correlates of motor intention and imaginery, Behavioral Brain Sciences, 17(1994).

Kant I., 2004, Kritik der praktischen Vernunft, Hrsg. von Wilhelm Weischedel, Suhrkamp, Frankfurt am Main.

Kant I., 2004, Kritik der Urteilskraft, Hrsg. von Wilhelm Weischedel, Suhrkamp, Frankfurt am Main.

Ingarden R., 1931, Das literarische Kunstwerk. Eine Untersuchung aus dem Grenzgebiet der Ontologie, Logik und Literaturwissenschaft, Max Niemeyer, Halle.

Ingarden R., 1968, Vom Erkennen des literarischen Kunstwerks, Max Niemeyer, Tübingen.

Patcherie E., Dokic J., 2006, From mirror neurons to joint actions, Cognitive Systems Research, 7(2006).

Rizzolatti G., Sinigaglia C., 2008, Empathie und Spiegelneurone. Die biologische Basis des Mitgefühls, Suhrkamp Verlag, Frankfurt am Main.

Russel B., 1905, On Denoting, Mind 14 (1905).

Stegmüller W., 1986, Die Sogenannte Methode des Verstehens, [in:] Lust an der Erkenntnis: Die Philosophie des 20. Jahrhunderts, Volker Spierling (Hrsg.), Serie Piper, München. 
Tokarz M., 1993, Elementy pragmatyki logicznej, PWN, Warszawa.

Urchs M., 2002, Maschine, Körper, Geist. Eine Einführung in die Kognitionswissenschaft, Vittorio Klostermann, Frankfurt am Main.

Wittgenstein L., 2003, Philosophische Untersuchungen, Suhrkamp Verlag, Frankfurt am Main.

\section{Abstract \\ Dimensions of Understanding}

In this paper I try to consider the question of understanding as a special mental capacity in the light of the neuroscientific approach and the philosophical tradition. I assert that it is not sufficient to explain the process of understanding on the subpersonal level. The understanding has several dimensions that are revealed only in the aspect of interpretation, communication and social interaction. This kind of understanding implies the meta-representational knowledge, which we wanted to present on the example of rational and hermeneutic thinking. It should be clear that in these cases, the reduction of holistic higher mental states gathered in the form of the mind theory to the neuronal level is not possible.

Key words: understanding, knowledge, pragmatics, mirror neurons, hermeneutic, rationality 